\title{
Treating highly complex anal fistula with a new method of combined intraoperative endoanal ultrasonography (IOEAUS) and transanal opening of intersphincteric space (TROPIS)
}

\author{
Baolei Huang, Xu Wang, Dongxu Zhou, Si Chen, Bai Li, Yilin Wang, Jiandong Tai \\ Department of Colorectal Surgery, the First Hospital of Jilin University, Changchun, China
}

Videosurgery Miniinv 2021; 16 (4): 697-703

DOI: https://doi.org/10.5114/wiitm.2021.104368

\begin{abstract}
Introduction: Treatment of highly complex anal fistula is still a profound test for a specialist colorectal surgeon. The reasons are directly related to recurrence and incontinence.

Aim: To evaluate the clinical results of a combined method of intraoperative endoanal ultrasonography (IOEAUS) and transanal opening of the intersphincteric space (TROPIS).

Material and methods: This study retrospectively included 48 patients with complex anal fistula, all of whom underwent new surgical methods. This operation mainly consists of two steps. Firstly, the type of anal fistula was determined by endoanal ultrasonography (EAUS) or magnetic resonance imaging (MRI) before the operation. Then the TROPIS procedure was performed with the help of EAUS, and the decision on whether a drainage seton should be placed depended on the condition of the tract. If there were secondary tracts, they were found and the same was done. Results: The median follow-up was 12 months. Two (4.1\%) patients experienced recurrence. Four (8.3\%) patients did not have primary healing. All 6 patients underwent the same procedure again, and three recovered completely. So total successful fistula healing was observed in 45 (93.7\%). There were no major complications and no significant deterioration in anal function and incontinence postoperatively.

Conclusions: Combined IOEAUS and TROPIS is an effective procedure in the treatment of highly complex anal fistula, and it may offer a new means for other operations.
\end{abstract}

Key words: anal fistula, fecal incontinence, recurrence, ultrasonography, transanal opening of intersphincteric space (TROPIS).

\section{Introduction}

The causes of anal fistula and abscess are various [1]. The estimated prevalence of nonspecific anal fistulas is 8.6 to $10 / 100,000$ of the population per year, with a male to female ratio of $1.8: 1$, and surgery is the main method to solve anal fistula [2]. Generally, the treatment of low anal fistula is relatively simple. A good recovery rate can be achieved by fistulotomy, and there is no difference in postop- erative anus function and recurrence rate compared with fistulectomy [3, 4]. In contrast, direct incision of a highly complex anal fistula increases the risk of postoperative fecal incontinence. Garg recently came up with a new standard about anal fistula [5], in which he advised that the lower grades (grades I-II) can conveniently undergo fistulectomy and grade III, IV and V should undergo sphincter-sparing procedures, and he confirmed the effectiveness of this classification in the subsequent study [6]. Therefore, 
correct preoperative judgment is an important prerequisite for solving highly complex anal fistula. It has been proved that anal magnetic resonance imaging (MRI) is more accurate than fistulography [7], and compared with the latter, MRI does not increase the risk of infection and does not cause any discomfort. Endoanal ultrasonography (EAUS) is of great help in the assessment of the position of the internal opening (IO), primary tract (PT), and presence of secondary tracts, and in the detection of associated anal sphincter (AS) defects [8]. Although some studies have shown that EAUS is less accurate than MRI scan [9], it is superior in its simplicity, economy and repeatability. It is worth noting that, based on those advantages, EAUS can be considered to be used in the operation of highly complex anal fistula to guide the operation process. There are no relevant reports and records in this regard at present.

Transanal opening of intersphincteric space (TROPIS) is a novel surgical technique described first by Garg [10]. This sphincter sparing procedure showed a $90.4 \%$ healing rate. It is performed by cutting the mucosa and internal sphincter in electrocautery from inside the rectum and curetted the tracts from the external opening [10]. However, we encountered some problems with our initial experiments. First of all, for this operation, it is most im-

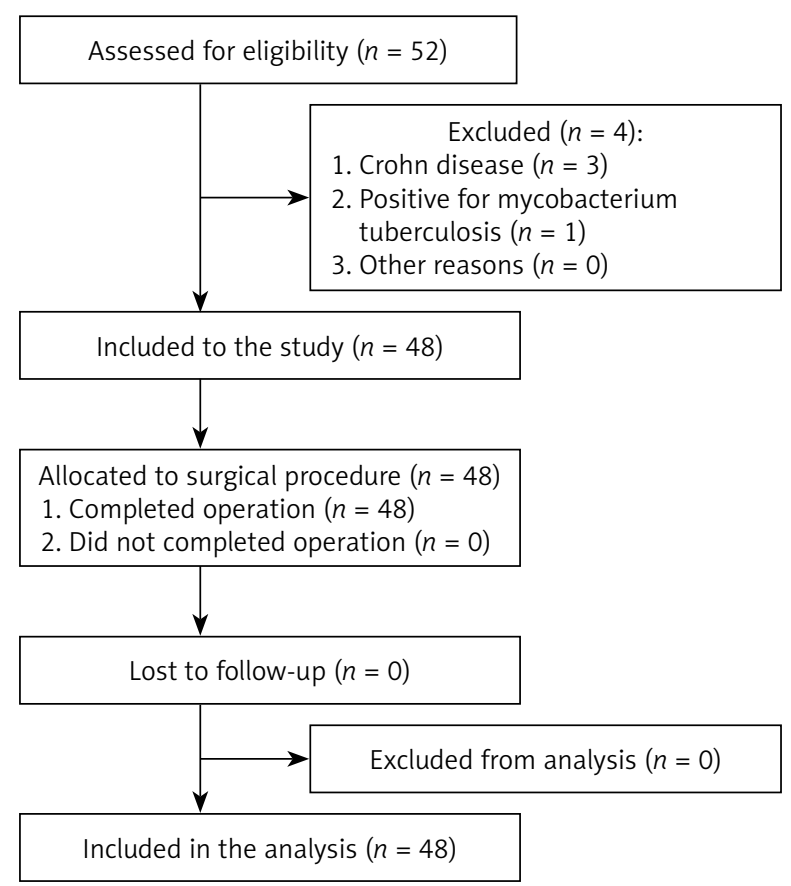

Figure 1. CONSORT flowchart showing the flow of patients through the trial portant to find the 10 correctly, but in some cases, 10 cannot be found accurately. Secondly, when scraping the external tracts, it is not easy to accurately identify some slender, curved ones or they may even be missed. Although the schematic diagram was made according to MRI before surgery, good feedback could not be obtained during the operating process. Finally, external tracts tend to heal earlier than internal incisions, leaving a dead space, which may lead to postoperative recurrence. Therefore, we took full advantage of EAUS to improve the recognition rate and the success rate of fistula by dynamically guiding the operation process in the operation. At the same time, for some slender and curved tracts, a drainage seton was adopted to facilitate postoperative cleaning and ensure the continuous emptying of the tracts during the whole healing process.

\section{Aim}

The aim of this study was to evaluate the clinical effect of a combined method of IOEAUS and TROPIS for highly complex anal fistula for healing while avoiding postoperative incontinence.

\section{Material and methods}

\section{Patients}

We retrospectively analyzed all patients with complex anal fistula who underwent combined IOEAUS and TROPIS surgery in the first hospital of Jilin University between October 2017 and December 2019. All the patients were informed of the course and the possible postoperative complications and signed informed consent for the operation. Institutional ethics committee approval was obtained. The flow chart of screening patients is shown in Figure 1.

Inclusion criteria: (1) highly complex anal fistula; (2) horseshoe fistulas; (3) supralevator fistula.

Exclusion criteria: (1) simple anal fistula; (2) patients with chronic illness affecting the healing process such as combined with immuno-compromised disease or related to Crohn's disease.

\section{Assessment}

Preoperative: All patients were examined at the outpatient clinic. Their medical history was examined in detail, including chief complaint, time of onset and duration, main symptoms, treatment history, and whether other diseases exist. After careful digi- 
tal rectal examination and endoscopy, patients were given EAUS or MRI to determine the type of the anal fistula, including the location of the external opening (EO) and IO, tracts, and to determine the relationship with levator ani muscle, and at the same time to exclude other lesions. Patients who met the inclusion criteria were graded with the Wexner incontinence score [11]. In addition, before the operation, professional operators used an anorectal pressure detector (model: ZGJ-D2) to measure anorectal pressure in the outpatient examination room.

Postoperative: (1) Postoperative discomfort. In addition to pain, more complex and strict dressing changes are often needed in the operative area for fecal contamination, incision secretion, and exudation, thus increasing the difficulty of postoperative care and the discomfort of the patients themselves. (2) Incontinence assessment: The Wexner incontinence score was measured 6 months after surgery and used to assess the postoperative patients' ability to control solid, liquid, and gas, as well as their lifestyle changes, the need to wear pads and so on. Each item is evaluated by a 5 -level scoring method with 0-4 points. The total score is scored from 0-20 points, with 0 being normal and 20 being total incontinence. The higher the score is, the more serious is the incontinence. Meanwhile, we measured the anal canal resting pressure and maximum contractive pressure. (3) Surgical success rate and recurrence rate: complete healing was defined as complete epithelialization of the wound, without signs of fistula external openings or perianal secretions. Postoperative follow-up showed that the operation failed in any of the following cases: anal fistula recurrence, recurrent abscess discharge, recheck MRI, or EAUS showed unhealed tract.

\section{Operative procedure}

All patients were given enemas of $500 \mathrm{ml}$ with $0.1 \%$ warm soapy water 2 to $3 \mathrm{~h}$ before the operation. If the patient still had a significant feeling of incomplete defecation after defecation, the same method of enema was performed again. And prophylactic antibiotics were given $0.5 \mathrm{~h}$ before surgery. After being admitted to the operating room, the patient was placed in a lithotomic position or prone jackknife position, and given general anesthesia, then fixed. The location and course of the anal fistula were determined again using EAUS. All ultrasound examina- tions were performed by the same radiologist, who brought a portable ultrasound diagnostic instrument (machine model: mylab-90; probe model: trt-33) into the operating room when the operation was needed and assisted the operation alongside the surgeon. With the guidance of EAUS, the position of the internal opening can be easily and accurately determined. A transparent anoscope was inserted into the anal canal to assist in the exposure. With 10 as the center, electrocautery was used to slide open the rectal mucosa and part of the internal anal sphincter in the direction of the fistula to expose the gap between the internal and external sphincter. For horseshoe fistulas or supra levator, the incision would be extended laterally or upward. The external opening was widened to $1.5-2.0 \mathrm{~cm}$, and through it a malleable metallic probe was inserted into the tract. With the help of EAUS, the probe was extended to the position of the internal opening, and the tract was fully cleaned with a curette to destroy the fistula epithelium. Finally the surgical field washed with saline and checked for hemostasis. The condition of the tract was assessed; if it was slender and curved, a drainage seton was placed. Throughout the operation, if there were secondary tracts or some other difficult situation to deal with, the perfect effect could be achieved by repeated use of EAUS.

\section{Postoperative management and follow-up}

Usually, patients were treated with antibiotics for 3 days after surgery. If examination of blood routine or rectal ultrasound indicated that inflammation was poorly controlled or even worse, prolonged treatment with antibiotics or a change of antibiotic type would be required. In all 48 patients, leukocyte and neutrophil levels increased in 2 patients after surgery and returned to normal after 7 days of prolonged antibiotic treatment. None of the patients had severe complications such as abscesses. The patients were discharged on the day after the operation. They were able to exercise normally on the same day and were encouraged to increase their daily walking volume to facilitate drainage. The dressing was changed every day for the first 3 days, the bleeding and seepage were observed, and a warm water bath was given before each dressing change. After that, the tracts would be cleaned with a curette every 2 days. After 10 days, we suggest that the patient's relatives learn the procedure so that they can change the 
dressing at home. Patients were followed up in the outpatient clinic once a week in the first month after surgery, then every other week, and the interval was gradually increased. The follow-up mainly includes the state of wound healing, recurrence, and complications. The mean time to complete healing was counted only for patients with primary healing. The anal function and continence state was evaluated by the anorectal pressure determination and Wexner incontinence score at 6 months postoperatively.

\section{Statistical analysis}

The relevant data in the study were input into the SPSS Statistics version 22 statistical package (IBM Corp., Armonk, NY, USA) for analysis. The counting data were represented by frequency (percentage, $\%)$, and the measurement data were represented by mean \pm standard deviation. Student's $t$-test was used to determine the significance of the difference in one group before and after the operation, and the $\chi^{2}$ test was used for enumeration data. $P<0.05$ was taken to indicate statistical significance.

\section{Results}

\section{Clinical characteristics of the patients}

Forty-eight patients with complex fistula-in-ano were retrospectively analyzed $(n=48)$, and the ba-

Table I. Patients' characteristics $(n=48)$

\begin{tabular}{|c|c|}
\hline Variable & Value \\
\hline Male/female & $41 / 7$ \\
\hline Mean age [years] & $40 \pm 11.7$ \\
\hline Multiple tracts, $n(\%)$ & $32(66.7)$ \\
\hline Horseshoe fistula, $n(\%)$ & $14(29.2)$ \\
\hline Associated/presented with abscess, $n(\%)$ & $29(60.4)$ \\
\hline Anterior tract, $n(\%)$ & $12(25.0)$ \\
\hline Internal opening not found, $n(\%)$ & $3(6.2)$ \\
\hline Supralevator extension, $n(\%)$ & $10(20.8)$ \\
\hline Recurren, $n(\%)$ : & $22(45.8)$ \\
\hline Incision + draining seton & $7(14.5)$ \\
\hline Incision + cutting seton & $1(0.2)$ \\
\hline Lay open & $14(29.1)$ \\
\hline \multicolumn{2}{|l|}{ Duration of prior treatment [months]: } \\
\hline Mean (SD) & $4.3(2.2)$ \\
\hline Range & $1-12$ \\
\hline
\end{tabular}

sic information and characteristics of the fistulas are summarized in Table I. The median age of the patients was $40 \pm 11.7$ years, 41 (85.4\%) patients were male and 7 (14.6\%) were female. The fistula characteristics were multiple tracts in 32 (66.7\%), recurrent in 22 (45.8\%), horseshoe in 14 (29.2\%), anterior tract in $12(25.0 \%)$, associated/presented with abscess in 29 (60.4\%) and 10 (20.8\%) patients had supralevator extension.

\section{Treatment}

The results of various types of anal fistula treated by this operation are shown in Table II. Of the 48 patients, fistula showed complete healing in $87.5 \%$ (42/48), 4 (8.3\%) failed to heal, and 2 (4.2\%) had recurrence. All the 6 patients who did not heal underwent a second operation with the first procedure, and 3 achieved complete healing, with no recurrence on further follow-up. Therefore, total

Table II. Types of anal fistula and healing rate $(n=48)$

\begin{tabular}{|c|c|c|c|}
\hline Variable & Number & Healed (\%) & $P$-value \\
\hline Multiple tracts: & & & 0.969 \\
\hline Multiple & 32 & $28(87.5)$ & \\
\hline Single & 16 & $14(87.5)$ & \\
\hline Recurrent: & & & 0.251 \\
\hline Recurrent & 22 & $18(81.2)$ & \\
\hline Primary & 26 & $24(92.3)$ & \\
\hline Horseshoe fistula: & & & 0.742 \\
\hline Horseshoe & 14 & $12(85.7)$ & \\
\hline Non-horseshoe & 34 & $30(88.2)$ & \\
\hline $\begin{array}{l}\text { Associated/presented } \\
\text { with abscess: }\end{array}$ & & & 0.613 \\
\hline Abscess & 29 & $26(89.7)$ & \\
\hline No-abscess & 19 & $16(84.2)$ & \\
\hline Anterior tract: & & & 0.543 \\
\hline Anterior & 12 & $10(83.3)$ & \\
\hline Non- anterior & 36 & $32(88.9)$ & \\
\hline $\begin{array}{l}\text { Internal opening not } \\
\text { found }\end{array}$ & 3 & $3(100)$ & \\
\hline $\begin{array}{l}\text { Supralevator exten- } \\
\text { sion }\end{array}$ & 10 & $7(70)$ & \\
\hline After 1 procedure & & $42(87.5)$ & \\
\hline $\begin{array}{l}\text { After } 2^{\text {nd }} \text { procedure in } \\
6 \text { patients }\end{array}$ & & $45(93.8)$ & \\
\hline
\end{tabular}


successful fistula healing occurred in 45 (93.8\%) Meanwhile, there was no statistically significant difference among subgroups ( $p>0.05)$.

Other surgical results and complications are shown in Table III. The mean operation time was 41 (32-75) min. The median follow-up time was 12 months, and no patients were lost. The mean time to complete healing was $7.24 \pm 1$ weeks. Four (8.3\%) patients had varying degrees of complications, of which $3(6.7 \%)$ had mild bleeding symptoms in about 7 days after surgery. One was controlled by suturing close the hemorrhage arteriole. The other two were controlled by manual pressure. One (2.1\%) showed urinary retention. The Visual Analog Scale (VAS) scores were used to assess postoperative pain in patients, with non-steroidal anti-inflammatory drugs (NSAIDs) given at lower levels and opioid analgesics given when poorly controlled. Some patients reported minor pain when changing the dressing. We gave these patients NSAIDs 1-2 times a day, which were well managed. None of the patients had severe pain.

The Wexner incontinence score, anal resting pressure and maximum anal contractive pressure before surgery did not show a significant difference $(p>0.05)$ compared with 6 months after surgery (Table IV).

\section{Discussion}

With the development of the medical treatment level, the single cure rate of anal fistula is no longer the only evaluation standard. How to effectively reduce the postoperative complications of anal fistula, maintain the normal physiological function of the anal sphincter, reduce postoperative pain and improve the quality of life is a hot topic in the surgical research of colorectal and anal surgery at present. According to the report of Garcia Aguillar, factors associated with recurrence included complex type of fistula, horseshoe extension, lack of recognition or lateral position of 10 , previous fistula surgery, and the surgeon operating. Incontinence was associated with female gender, high anal fistula, type of surgery, and previous fistula surgery [12]. Jordán et al.,
Table III. Other surgical results and complications $(n=48)$

\begin{tabular}{|lc|}
\hline Parameter & Result \\
\hline Mean time to complete healing [weeks] & $7.24 \pm 1$ \\
\hline Failed healing & $4(8.3 \%)$ \\
\hline Recurrence & $2(4.2 \%)$ \\
\hline Median recurrence time [months] & 6 \\
\hline Complications & $4(8.3 \%)$ \\
\hline Bleeding & $3(6.7 \%)$ \\
\hline Urinary retention & $1(2.1 \%)$ \\
\hline Severe pain & $0(0 \%)$ \\
\hline Fecal incontinence & $0(0 \%)$ \\
\hline Mean operative time [min] & $41(32-75)$ \\
\hline Median follow-up [months] & 12 \\
\hline
\end{tabular}

on the other hand, reported only complex fistula and nonidentification of 10 was statistically significant in recurrence and only previous incontinence as a risk factor of incontinence [13]. In either outcome, the treatment of complex anal fistula, especially identification of the $\mathrm{IO}$ and tract, is particularly important. IOEAUS can play a big role here. IOEAUS has four main advantages: to obtain new information that cannot be obtained by other methods, to supplement or replace intraoperative imaging, to guide the surgical procedure, and to confirm the completion of the surgery. Compared with intraoperative angiography, IOU also has the advantages of safety, fast speed, high accuracy, more information acquisition, and wide application. Above all, and in combination with some other research data $[8,14]$, we suggest that only EAUS examination could be performed before surgery, and MRI supplementation can be selected if necessary.

According to our study, the primary healing rate and the total healing rate were higher than in the report of Pankaj Garg, and the recurrence rate was lower. Four patients failed to show primary healing, and 2 patients experienced recurrence; we ascribe the reason to more use of a drainage seton.

Table IV. Comparison of Wexner incontinence score, anorectal pressure before and after the operation

\begin{tabular}{|lcccc|}
\hline Variable & Preoperative & Postoperative & $P$-value & $t$ \\
\hline Wexner incontinence score & $0($ range: $0-20)$ & $0($ range: $0-20)$ & 0 & - \\
\hline Anal resting pressure $[\mathrm{mm} \mathrm{Hg}]$ & $64.56 \pm 8.590$ & $63.44 \pm 7.370$ & 0.06 & 1.90 \\
\hline Anal contractive pressure $[\mathrm{mm} \mathrm{Hg}]$ & $141.79 \pm 8.906$ & $140.67 \pm 7.969$ & 0.10 & 1.70 \\
\hline
\end{tabular}


The cutting seton is known to increase the risk of incontinence [15]. So the purpose of the seton we used was not to incise the sphincter, but to allow for the tract to be kept open and easy to clean. Such a good-drainage environment was more favorable for rectal incision healing and removing the seton when the internal opening was healed. However, it also brought its issues. If the seton was removed too early, serous secretions and blood might flow back and accumulate in the internal incision, resulting in non-healing and even infection. If the seton was removed too late, secondary fistula and cavity would be formed, leaving the risk of recurrence. Therefore, we recommend that EAUS be conducted twice before the seton is removed to ensure optimal timing. Specific criteria need a long time to be confirmed.

None of the patients complained of postoperative fecal incontinence. The anorectal pressure determination also confirmed that our procedure can well retain the function of the anus, because the external anal sphincter is retained and partial internal sphincter is destroyed. A small number of patients reported mild leakage in the first few days after surgery, but it quickly disappeared. According to the results of subsequent telephone follow-up, $90 \%$ of the patients were satisfied with the treatment effect and postoperative quality of life, while the remaining $10 \%$ reported mild pain or seton use or secondary surgery. In general, our surgical approach is designed to ensure a high rate of healing, a low rate of recurrence, and at the same time to ensure as much quality of life as possible.

In recent years, procedures of sphincter sparing have become increasingly rich, including improvements to traditional surgical and innovations from new methods, the most famous of which are ligation of the intersphincteric fistula tract (LIFT) and assisted anal fistula treatment (VAAFT).

Inspired by the clinical application of cystoscopy, Meinero et al. [16] first proposed VAAFT in 2011. The main method is to insert the anal fistuloscope through the external opening, probe the fistula condition, find the correct internal opening, and then destroy and remove the necrotic fistula tissue by electric cauterization, scratching, rinsing, etc., and close the internal opening with the stapler or skin mucous flap. However, VAAFT does not resolve the intersphincteric abscess well and remains continuously empty, with the most recent MATA analysis show- ing an average recurrence rate of around 18\% [17]. At the same time, VAAFT also has some problems such as high cost and difficulty in the treatment of fistula curvature and narrow or horseshoe anal fistula. In addition, compared with the complex learning curve of the anal fistuloscope, EAUS is easier to learn.

In 2007, Rojanasakul et al. [18] proposed LIFT for the first time. In contrast to TROPIS, LIFT also involves the processing of the intersphincteric tract, but with the internal opening closed. However, accurate identification of fistulas and complete fistula separation require a considerable technical level, especially for long or highly complex anal fistula. Liu et al. [19] reported an overall primary healing rate of $61 \%$. Although there are some improvements and innovations to LIFT, such as the use of bioprosthetic grafts to reinforce the ligation of the intersphincteric fistula tract (BioLIFT) [20] and ligation of the inter-sphincteric fistula tract plus bioprosthetic anal fistula plug (LIFT-Plug) [21], which have a cure rate of $94 \%$ and $95 \%$, respectively, they cannot be widely used in the clinic due to the high cost. Therefore, compared with LIFT, TROPIS is a better choice for highly complex anal fistula.

Recently, Bobkiewicz et al. proposed a novel concept for the treatment of complex anal fistula, namely endoscopic vacuum therapy with instillation (iEVT) [22]. It is a combination of standard endoscopic vacuum therapy and negative pressure wound therapy (NPWT) with instillation (iNPWT). This is a minimally invasive procedure and, in some cases, can be used as a transitional therapy prior to surgery. A larger sample size and longer follow-up time are needed to determine the effectiveness of the technique.

Additionally, there are some other surgical methods to treat highly complex anal fistula, such as expanded adipose-derived stem cells (ASCs) [23], dermal collagen injection [24], fibrin sealant [25], FiLaC [26], etc., which have achieved good therapeutic effects, but the specific role still needs to be clarified in more randomized trials.

Although our surgery has increased steps and time relative to the original TROPIS, the benefits to patients are predictable. At the same time, we believe that IOEAUS, of which EAUS is not only used for diagnosis but also treatment, provides a new means for other procedures and helps them to improve the operation.

This study has some limitations, mainly that this is a single-center study, not a multi-center random- 
ized controlled trial, and had selection bias; the review was followed up for 12 months and the number of patients was 48. Longer time and larger sample sizes are needed for a more accurate evaluation of effectiveness.

\section{Conclusions}

Combined TROPIS and IOEAUS are effective in the treatment of highly complex anal fistula, with a low recurrence rate while maintaining good cure rates and anal function, and it may offer a new means for other operations.

\section{Acknowledgments}

Baolei Huang and Xu Wang contributed equally to this work.

\section{Conflict of interest}

The authors declare no conflict of interest.

\section{References}

1. Amato A, Bottini C, De Nardi P, et al. Evaluation and management of perianal abscess and anal fistula: SICCR position statement. Tech Coloproctol 2020; 24: 127-43.

2. Ratto C, Grossi U, Litta F, et al. Contemporary surgical practice in the management of anal fistula: results from an international survey. Tech Coloproctol 2019; 23: 729-41.

3. Kronborg O. To lay open or excise a fistula-in-ano: a random ized trial. Br J Surg 1985; 72: 970

4. Anan M, Emile SH, Elgendy H, et al. Fistulotomy with or with out marsupialisation of wound edges in treatment of simple anal fistula: a randomised controlled trial. Ann R Coll Surg Eng 2019; 101: 472-8.

5. Garg P. Comparing existing classifications of fistula-in-ano in 440 operated patients: is it time for a new classification? A Retrospective Cohort Study. Int J Surg 2017; 42: 34-40.

6. Garg P. Is fistulotomy still the gold standard in present era and is it highly underutilized? An audit of 675 operated cases. Int J Surg 2018; 56: 26-30.

7. Balcı S, Onur MR, Karaosmanoğlu AD, et al. MRI evaluation of anal and perianal diseases. Diagn Interv Radiol 2019; 25: 21-7.

8. Almeida IS, Jayarajah U, Wickramasinghe DP, et al. Value of three-dimensional endoanal ultrasound scan (3D-EAUS) in preoperative assessment of fistula-in-ano. BMC Res Notes 2019 $12: 66$

9. Brillantino A, lacobellis F, Reginelli A, et al. Preoperative assessment of simple and complex anorectal fistulas: tridimensional endoanal ultrasound? Magnetic resonance? Both? Radiol Med 2019; 124: 339-49.

10. Garg P. Transanal opening of intersphincteric space (TROPIS) a new procedure to treat high complex anal fistula. Int J Surg 2017; 40: 130-4.
11. Jorge MJN, Wexner SD. Etiology and management of fecal incontinence. Dis Colon Rectum 1993; 36: 77-97.

12. Garcia-Aguilar J, Belmonte C, Wong WD, et al. Anal fistula surgery. Factors associated with recurrence and incontinence. Dis Colon Rectum 1996; 39: 723-9.

13. Jordán J, Roig JV, García-Armengol J, et al. Risk factors for recurrence and incontinence after anal fistula surgery. Colorectal Dis 2010; 12: 254-60.

14. Kołodziejczak M, Santoro GA, Obcowska A, et al. Three-dimensional endoanal ultrasound is accurate and reproducible in determining type and height of anal fistulas. Colorectal Dis 2017; 19: 378-84.

15. Andreou C, Zeindler J, Oertli D, et al. Longterm outcome of anal fistula - a retrospective study. Sci Rep 2020; 10: 6483.

16. Meinero P, Mori L. Video-assisted anal fistula treatment (VAAFT): a novel sphincter-saving procedure for treating complex anal fistulas. Tech Coloproctol 2011; 15: 417-22.

17. Emile SH, Elfeki $H$, Shalaby $M$, et al. A Systematic review and meta-analysis of the efficacy and safety of video-assisted anal fistula treatment (VAAFT). Surg Endosc 2018; 32: 2084-93.

18. Rojanasakul A, Pattanaarun J, Sahakitrungruang C, et al. Total anal sphincter saving technique for fistula-in-ano; the ligation of intersphincteric fistula tract. J Med Assoc Thai 2007; 90: 581-6.

19. Liu WY, Aboulian A, Kaji AH, et al. Long-term results of ligation of intersphincteric fistula tract (LIFT) for fistula-in-ano. Dis CoIon Rectum 2013; 56: 343-7.

20. Ellis CN. Outcomes with the use of bioprosthetic grafts to reinforce the ligation of the intersphincteric fistula tract (BioLIFT procedure) for the management of complex anal fistulas. Dis Colon Rectum 2010; 53: 1361-4.

21. Zhao B, Wang Z, Han J, et al. Long-term outcomes of ligation of the inter-sphincteric fistula tract plus bioprosthetic anal fistula plug (LIFT-Plug) in the treatment of trans-sphincteric perianal fistula. Med Sci Monit 2019; 25: 1350-4.

22. Bobkiewicz A, Krokowicz L, Banasiewicz T, et al. Endoscopic vacuum therapy with instillation (iEVT) - a novel endoscopic concept for colorectal anastomotic leak and perianal complications. Videosurgery Miniinv 2020; 15: 560-6.

23. Garcia-Olmo D, Herreros D, Pascual I, et al. Expanded adipose-derived stem cells for the treatment of complex perianal fistula: a phase II clinical trial. Dis Colon Rectum 2009; 52: 79-86.

24. Himpson RC, Cohen CRG, Sibbons P, et al. An experimentally successful new sphincter-conserving treatment for anal fistula. Dis Colon Rectum 2009; 52: 602-8.

25. Buchanan GN, Bartram Cl, Phillips RK, et al. Efficacy of fibrin sealant in the management of complex anal fistula: a prospective trial. Dis Colon Rectum 2003; 46: 1167-74.

26. Wilhelm A. A new technique for sphincter-preserving anal fistula repair using a novel radial emitting laser probe. Tech Coloproctol 2011; 15: 445-9.

Received: 1.11.2020, accepted: 26.01.2021. 